\title{
REGULARITY AND FRACTAL DIMENSION OF PULLBACK ATTRACTORS FOR A NON-AUTONOMOUS SEMILINEAR DEGENERATE PARABOLIC EQUATION
}

\author{
CUNG THE ANH \\ Department of Mathematics, Hanoi National University of Education, \\ 136 Xuan Thuy, Cau Giay, Hanoi, Vietnam \\ e-mail: anhctmath@hnue.edu.vn \\ TANG QUOC BAO \\ Faculty of Applied Mathematics and Informatics, Hanoi University of Science and Technology, \\ 1 Dai Co Viet, Hai Ba Trung, Hanoi, Vietnam \\ e-mail:mathizonewww@gmail.com \\ and LE THI THUY \\ Department of Mathematics, Hanoi Electric Power University, \\ 235 Hoang Quoc Viet, Tu Liem, Hanoi, Vietnam \\ e-mail: thuylephuong@gmail.com
}

(Received 28 November 2011; accepted 25 July 2012; first published online 25 February 2013)

Abstract. Considered here is the pullback attractor of the process associated with the first initial boundary value problem for the non-autonomous semilinear degenerate parabolic equation

$$
u_{t}-\operatorname{div}(\sigma(x) \nabla u)+f(u)=g(x, t)
$$

in a bounded domain $\Omega$ in $\mathbb{R}^{N}(N \geq 2)$. We prove the regularity in the space $L^{2 p-2}(\Omega) \cap$ $\mathcal{D}_{0}^{2}(\Omega, \sigma)$, and estimate the fractal dimension of the pullback attractor in $L^{2}(\Omega)$.

2010 Mathematics Subject Classification. 37L30, 35B41, 35K65, 35D30.

1. Introduction. This paper is a continuation of the paper [2] in which we studied the long-time behaviour of solutions to the following non-autonomous degenerate parabolic equation:

$$
\left\{\begin{array}{l}
u_{t}-\operatorname{div}(\sigma(x) \nabla u)+f(u)=g(x, t), x \in \Omega, t>\tau \\
\left.u\right|_{\partial \Omega}=0 \\
\left.u\right|_{t=\tau}=u_{\tau} \in L^{2}(\Omega)
\end{array}\right.
$$

where $\Omega$ is a bounded domain in $\mathbb{R}^{N}(N \geq 2)$ with smooth boundary $\partial \Omega$, the diffusion coefficient $\sigma$, the nonlinearity $f$ and the external force $g$ satisfying the following conditions:

(H1) $\sigma$ is a non-negative measurable function such that $\sigma \in L_{\text {loc }}^{1}(\Omega)$ and for some $\alpha \in(0,2), \liminf _{x \rightarrow z}|x-z|^{-\alpha} \sigma(x)>0$ for every $z \in \bar{\Omega}$ 
(H2) The function $f \in C^{1}(\mathbb{R}, \mathbb{R})$ satisfies

$$
\begin{gathered}
f(u) u \geq C_{1}|u|^{p}-C_{2}, p \geq 2, \\
\left|f^{\prime}(u)\right| \leq C_{3}|u|^{p-2}, \\
f^{\prime}(u) \geq-\ell,
\end{gathered}
$$

where $\ell, C_{i}, i=1,2,3$ are positive constants. Relations (1.2) and (1.3) implies that

$$
\alpha_{1}|u|^{p}-\alpha_{2} \leq F(u) \leq \alpha_{3}|u|^{p}+\alpha_{4},
$$

where $F(u)=\int_{0}^{u} f(s) d s$, and $\alpha_{i}, i=1,2,3,4$, are positive constants;

(H3) $g \in W_{\text {loc }}^{1,2}\left(\mathbb{R} ; L^{2}(\Omega)\right)$ satisfies

$$
\int_{-\infty}^{0} e^{\lambda_{1} s}\left(\|g(s)\|_{L^{2}(\Omega)}^{2}+\left\|g^{\prime}(s)\right\|_{L^{2}(\Omega)}^{2}\right) d s<+\infty,
$$

where $\lambda_{1}>0$ is the first eigenvalue of the operator $-\operatorname{div}(\sigma(x) \nabla \cdot)$ in $\Omega$ with the homogeneous Dirichlet boundary condition (see Section 2 ).

Problem (1.1) can be derived as a simple model for neutron diffusion (feedback control of nuclear reactor) (see [5]). In this case $u$ and $\sigma$ stand for the neutron flux and neutron diffusion respectively. As mentioned in [2], the degeneracy of problem (1.1) is considered in the sense that the measurable, non-negative diffusion coefficient $\sigma(\cdot)$ is allowed to have at most a finite number of (essential) zeroes at some points. For the physical motivation of assumption $(\mathrm{H} 1)$, we refer the reader to $[3,6,7]$.

Let us give some comments about assumptions (H2)-(H3). The nonlinearity $f$ is assumed to satisfy a polynomial-type growth and a standard dissipative condition. It is noted that we make assumption (1.3) for the sake of brevity in the proofs only. In fact, one can assume that $f$ satisfies $\left|f^{\prime}(u)\right| \leq C_{3}|u|^{p-2}+C_{4}$, where $C_{3}$ and $C_{4}$ are positive constants. Typical examples of functions satisfying condition $(\mathrm{H} 2)$ are polynomials with odd degrees and positive leading coefficients. The conditions in (H3) hold if $g \in W_{\text {loc }}^{1,2}\left(\mathbb{R} ; L^{2}(\Omega)\right)$ and there exist $\gamma \in\left(0, \lambda_{1}\right), \tau \in \mathbb{R}$ (without loss of generality, we can assume $\tau<0)$ and $M_{\tau}>0$ such that $\|g(t)\|_{L^{2}(\Omega)}^{2}+\left\|g^{\prime}(t)\right\|_{L^{2}(\Omega)}^{2} \leq M_{\tau} e^{-\gamma t}$ for all $t \leq \tau$. In particular, condition (H3) holds if $\|g(t)\|_{L^{2}(\Omega)}^{2}+\left\|g^{\prime}(t)\right\|_{L^{2}(\Omega)}^{2} \leq M e^{\gamma|t|}$ for all $t \in \mathbb{R}$. In the paper [2], under certain conditions which are similar to (H1)-(H3), the authors proved the existence of a pullback attractor in the space $\mathcal{D}_{0}^{1}(\Omega, \sigma) \cap L^{p}(\Omega)$ for the process $U(t, \tau)$ associated to problem (1.1). Here and what follows, for definitions of the function spaces related to problem (1.1), we refer reader to Section 2.1.

In this paper, under some additional conditions of the external force $g$, namely,

$$
\begin{aligned}
& \text { (H4) } \int_{-\infty}^{0} e^{\lambda_{1} s}\left\|g^{\prime}(s)\right\|_{L^{m_{k}(\Omega)}}^{m_{k}^{\prime}} d s<+\infty, \text { where } m_{k}=\frac{2 \beta^{k+2}}{2 \beta^{k+2}+1-2 \beta^{k+1}}, m_{k}^{\prime}=2 \beta^{k+1}, \text { with } \\
& \beta=\frac{N}{N-2+\alpha}>1, k \in \mathbb{N}, 0 \leq k \leq \log _{\beta}(p-1)+1,
\end{aligned}
$$

we will show that the pullback attractor obtained in [2] is in fact in $\left.\mathcal{D}_{0}^{2}(\Omega, \sigma) \cap L^{2 p-2} \Omega\right)$, that is, it is more regular. To do this, we first establish the existence of a family of pullback absorbing sets in $L^{2 p-2}(\Omega) \cap \mathcal{D}_{0}^{2}(\Omega, \sigma)$ and then prove the pullback 
asymptotic compactness of the process $U(t, \tau)$ in $L^{2 p-2}(\Omega) \cap \mathcal{D}_{0}^{2}(\Omega, \sigma)$. The proof of the first one is quite standard, but the proof of the second one is more involved. Indeed, as we know, if the external force $g(t)$ is only in $L^{2}(\Omega)$, then solutions $u(t)$ of problem (1.1) are at most in $L^{2 p-2}(\Omega) \cap \mathcal{D}_{0}^{2}(\Omega, \sigma)$ and have no higher regularity. Therefore, there is no compact embedding result held for this case. To overcome the difficulty caused by the lack of compactness of embeddings, we exploit the asymptotic a priori estimate method introduced in [14] for autonomous equations. The asymptotic a priori estimates in $L^{2 p-2}(\Omega)$ are deduced by using some estimates on $u_{t}$ that are based on assumption (H4) of the external force $g$ (see Lemma 3.3), and the pullback asymptotic compactness in $\mathcal{D}_{0}^{2}(\Omega, \sigma)$ is proved by verifying the (PDC) condition introduced in [9]. As a result, we obtain the existence of pullback attractors in the spaces $L^{2 p-2}(\Omega)$ and $\mathcal{D}_{0}^{2}(\Omega, \sigma)$. These pullback attractors and the pullback attractor obtained in [2] are of course the same object. The obtained result seems to be optimal, and, in particular, we obtain the $\mathcal{D}_{0}^{2}(\Omega, \sigma) \cap L^{2 p-2}(\Omega)$-boundedness of the pullback attractor. Using this boundedness, we show that the pullback attractor has a finite fractal dimension in $L^{2}(\Omega)$. It is worth noticing that when $\sigma=1$, we extend and improve the recent results on pullback attractors for the non-autonomous reaction-diffusion equations in bounded domains (see, e.g. $[1,9,10,12,13])$. Moreover, (H3) and (H4) are obviously satisfied if $g=g(x) \in L^{2}(\Omega)$ does not depend on time $t$, and then the pullback attractor turns to be the usual global attractor; therefore, we also recover some results in [14] when $\sigma=1$.

The paper is organized as follows. In Section 2, for convenience of the reader, we recall some concepts and results on function spaces and pullback attractors which we will use. In Section 3, we prove the existence of pullback attractors in various spaces by using the asymptotic a priori estimate method. In Section 4, we estimate the fractal dimension of the pullback attractor in $L^{2}(\Omega)$. Some further remarks are given in the last section.

\section{Preliminaries.}

2.1. Function spaces and operators. To study problem (1.1), we use the energy space $\mathcal{D}_{0}^{1}(\Omega, \sigma)$, defined as the closure of $C_{0}^{\infty}(\Omega)$ with respect to the norm

$$
\|u\|_{\mathcal{D}_{0}^{1}(\Omega, \sigma)}:=\left(\int_{\Omega} \sigma(x)|\nabla u|^{2} d x\right)^{\frac{1}{2}} .
$$

This is a Hilbert space with the scalar product

$$
(u, v):=\int_{\Omega} \sigma(x) \nabla u \nabla v d x .
$$

The following lemma comes from [3, Propositions 3.3-3.5].

Lemma 2.1. Assume that $\Omega$ is a bounded domain in $\mathbb{R}^{N}(N \geq 2)$, and $\sigma$ satisfies (H1). Then the following embeddings hold:

(i) $\mathcal{D}_{0}^{1}(\Omega, \sigma) \hookrightarrow L^{2_{\alpha}^{*}}(\Omega)$ continuously;

(ii) $\mathcal{D}_{0}^{1}(\Omega, \sigma) \hookrightarrow L^{p}(\Omega)$ compactly if $p \in\left[1,2_{\alpha}^{*}\right)$,

where $2_{\alpha}^{*}=\frac{2 N}{N-2+\alpha}$. 
We also use the space $\mathcal{D}_{0}^{2}(\Omega, \sigma)$ defined as the closure of $C_{0}^{\infty}(\Omega)$ with the norm

$$
\|u\|_{\mathcal{D}_{0}^{2}(\Omega, \sigma)}=\left(\int_{\Omega}|\operatorname{div}(\sigma(x) \nabla u)|^{2} d x\right)^{1 / 2} .
$$

The following result follows from the definitions of $\mathcal{D}_{0}^{1}(\Omega, \sigma)$ and $\mathcal{D}_{0}^{2}(\Omega, \sigma)$.

LEMma 2.2. If $\sigma$ satisfies $(H 1)$, then $\mathcal{D}_{0}^{2}(\Omega, \sigma) \subset \mathcal{D}_{0}^{1}(\Omega, \sigma)$ continuously.

Proof. For any function $u \in C_{0}^{\infty}(\Omega)$, we have

$$
\begin{aligned}
\|u\|_{\mathcal{D}_{0}^{1}(\Omega)}^{2}=\int_{\Omega} \sigma|\nabla u|^{2} d x & =-\int_{\Omega} \operatorname{div}(\sigma \nabla u) u d x \\
& \leq\left(\int_{\Omega}|\operatorname{div}(\sigma \nabla u)|^{2} d x\right)^{1 / 2}\left(\int_{\Omega}|u|^{2} d x\right)^{1 / 2}=\|u\|_{\mathcal{D}_{0}^{2}(\Omega)}\|u\|_{L^{2}(\Omega)} .
\end{aligned}
$$

Noticing that $\|u\|_{L^{2}(\Omega)} \leq C\|u\|_{\mathcal{D}_{0}^{1}(\Omega)}$, where $C$ is independent of $u$, we get the desired result from the fact that $C_{0}^{\infty}(\Omega)$ is dense in $\mathcal{D}_{0}^{2}(\Omega, \sigma)$.

It is known (see e.g. [2]) that the operator $A u:=-\operatorname{div}(\sigma(x) \nabla u)$ with the homogeneous Dirichlet boundary condition in $\Omega$ has a family $\left\{e_{n}\right\}_{n=1}^{\infty}$ of eigenvectors, which forms an orthonormal basis of $L^{2}(\Omega)$, and a sequence of eigenvalues $\left\{\lambda_{n}\right\}_{n \geq 1}$ such that $0<\lambda_{1} \leq \ldots \leq \lambda_{n} \leq \ldots$ and $\lambda_{n} \rightarrow+\infty$ as $n \rightarrow+\infty$. In the sequel, we frequently use the following inequality:

$$
\|u\|_{\mathcal{D}_{0}^{1}(\Omega, \sigma)}^{2} \geq \lambda_{1}\|u\|_{L^{2}(\Omega)}^{2}, \text { for all } u \in \mathcal{D}_{0}^{1}(\Omega, \sigma) .
$$

2.2. Pullback attractors. Let $X$ be a Banach space with the norm $\|\cdot\|$, and let $\mathcal{B}(X)$ denote all bounded sets of $X$. The Hausdorff semi-distance between two bounded subsets $A$ and $B$ of $X$ is defined by

$$
\operatorname{dist}(A, B)=\sup _{x \in A} \inf _{y \in B}\|x-y\| .
$$

Let $\{U(t, \tau): t \geq \tau, \tau \in \mathbb{R}\}$ be a process in $X$, i.e. $U(t, \tau): X \rightarrow X$ such that $U(\tau, \tau)=$ $I d$ and $U(t, s) U(s, \tau)=U(t, \tau)$ for all $t \geq s \geq \tau, \tau \in \mathbb{R}$. The process $\{U(t, \tau)\}$ is said to be norm-to-weak continuous if $U(t, \tau) x_{n} \rightarrow U(t, \tau) x$, as $x_{n} \rightarrow x$ in $X$, for all $t \geq$ $\tau, \tau \in \mathbb{R}$. The following result is useful for proving the norm-to-weak continuity of a process.

Proposition 2.3 [14]. Let $X, Y$ be two Banach spaces, $X^{*}, Y^{*}$ be respectively their dual spaces. Suppose that $X$ is dense in $Y$, the injection $i: X \rightarrow Y$ is continuous and its adjoint $i^{*}: Y^{*} \rightarrow X^{*}$ is dense, and $\{U(t, \tau)\}$ is a continuous or weak continuous process on $Y$. Then $\{U(t, \tau)\}$ is norm-to-weak continuous on $X$ if and only if for $t \geq \tau, \tau \in \mathbb{R}$, $U(t, \tau)$ maps compact sets of $X$ to be bounded sets of $X$.

Definition 2.1. The process $\{U(t, \tau)\}$ is said to be pullback asymptotically compact if for any $t \in \mathbb{R}$, any $D \in \mathcal{B}(X)$, any sequence $\tau_{n} \rightarrow-\infty$ and any sequence $\left\{x_{n}\right\} \subset D$, the sequence $\left\{U\left(t, \tau_{n}\right) x_{n}\right\}$ is relatively compact in $X$. 
Definition 2.2. A family of bounded sets $\mathcal{B}=\{B(t): t \in \mathbb{R}\} \subset X$ is called a family of pullback absorbing sets for the process $\{U(t, \tau)\}$ if for any $t \in \mathbb{R}$, any $D \in \mathcal{B}(X)$, there exists $\tau_{0}=\tau_{0}(D, t) \leq t$ such that

$$
\bigcup_{\tau \leq \tau_{0}} U(t, \tau) D \subset B(t) .
$$

Definition 2.3. A family $\mathcal{A}=\{A(t): t \in \mathbb{R}\} \subset \mathcal{B}(X)$ is said to be a pullback attractor for the process $\{U(t, \tau)\}$ if

(1) $A(t)$ is compact for all $t \in \mathbb{R}$,

(2) $\mathcal{A}$ is invariant, i.e.

$$
U(t, \tau) A(\tau)=A(t), \text { for all } t \geq \tau,
$$

(3) $\mathcal{A}$ is pullback attracting, i.e.

$$
\lim _{\tau \rightarrow-\infty} \operatorname{dist}(U(t, \tau) D, A(t))=0, \text { for all } D \in \mathcal{B}(X), \text { and all } t \in \mathbb{R},
$$

(4) if $\{C(t): t \in \mathbb{R}\}$ is another family of closed pullback attracting sets, then $A(t) \subset$ $C(t)$ for all $t \in \mathbb{R}$.

THEOREM 2.4 [9]. Let $\{U(t, \tau)\}$ be a norm-to-weak continuous process which is pullback asymptotically compact. If there exists a family of pullback absorbing sets $\mathcal{B}=\{B(t): t \in \mathbb{R}\}$, then $\{U(t, \tau)\}$ has a unique pullback attractor $\mathcal{A}=\{A(t): t \in \mathbb{R}\}$ and

$$
A(t)=\bigcap_{s \leq t} \overline{\bigcup_{\tau \leq s} U(t, \tau) B(\tau) .}
$$

2.3. Fractal dimension of pullback attractors. Given a compact $K \subset L^{2}(\Omega)$, and $\varepsilon>0$, we denote by $N_{\varepsilon}(K)$ the minimum number of open balls in $L^{2}(\Omega)$ with radii $\varepsilon$ that are necessary to cover $K$.

Definition 2.4. For any non-empty compact $K \subset L^{2}(\Omega)$, the fractal dimension of $K$ is the number

$$
d_{F}(K)=\limsup _{\varepsilon \downarrow 0} \frac{\log N_{\varepsilon}(K)}{\log (1 / \varepsilon)} .
$$

Firstly, we recall the definition of a uniform pullback absorbing set.

Definition 2.5 [8]. A bounded subset $B_{0}$ of $L^{2}(\Omega)$ is called a uniform pullback absorbing set for the process $\{U(t, \tau)\}$ if for every $B \in \mathcal{B}\left(L^{2}(\Omega)\right)$ there exists a number $\tau_{0} \geq 0$ such that

$$
U(t, t-\tau) B \subset B_{0}, \text { for all } \tau \geq \tau_{0},
$$

where $\tau_{0}$ does not depend on the choice of $t$.

Next, we describe the method to estimate the fractal dimension of a pullback attractor. 
THEOREM 2.5 [8]. Let $B_{0}$ be a uniform pullback absorbing set, $\mathcal{A}=\{A(t): t \in \mathbb{R}\}$ be a pullback attractor for $\{U(t, \tau)\}$. Assume that there exists a finite dimensional projection $P$ in the space $L^{2}(\Omega)$ such that

$$
\left.\| P\left(U\left(t, t-T_{0}\right) u_{1}\right)-U\left(t, t-T_{0}\right) u_{2}\right)\left\|_{L^{2}(\Omega)} \leq l\left(T_{0}\right)\right\| u_{1}-u_{2} \|_{L^{2}(\Omega)}
$$

for all $u_{1}, u_{2} \in B_{0}$ and some $T_{0}, l\left(T_{0}\right)$, and

$$
\left.\|(I-P)\left(U\left(t, t-T_{0}\right) u_{1}\right)-U\left(t, t-T_{0}\right) u_{2}\right)\left\|_{L^{2}(\Omega)} \leq \delta\right\| u_{1}-u_{2} \|_{L^{2}(\Omega)}
$$

for all $u_{1}, u_{2} \in B_{0}$, where $\delta<1$, and $T_{0}, l\left(T_{0}\right)$ are independent of the choice of $t$. Then the pullback attractor $\mathcal{A}$ possesses a finite fractal dimension in $L^{2}(\Omega)$, specifically

$$
\operatorname{dim}_{F}(A(t)) \leq \operatorname{dim} P \log \left(1+\frac{8 l\left(T_{0}\right)}{1-\delta}\right)\left[\log \frac{2}{1+\delta}\right]^{-1}, \quad \forall t \in \mathbb{R} .
$$

In the rest of the paper, for brevity, we denote by $|\cdot|_{2},(.,$.$) the norm and inner$ product in $L^{2}(\Omega)$. For a Banach space $E$, we denote by $\|\cdot\|_{E}$ the norm of $E$. A generic constant is denoted by $C$.

3. Existence of pullback attractors in $L^{2 p-2}(\Omega)$ and $\mathcal{D}_{0}^{2}(\Omega, \sigma)$. Under conditions $(\mathrm{H} 1)-(\mathrm{H} 3)$, by using arguments in $[\mathbf{2}, \mathbf{1 0}]$, it can be proved that one can define a process $\{U(t, \tau)\}_{t \geq \tau}$ associated to problem (1.1) as follows:

$$
U(t, \tau): L^{2}(\Omega) \rightarrow \mathcal{D}_{0}^{1}(\Omega, \sigma) \cap L^{p}(\Omega), \forall t \geq \tau,
$$

where $U(t, \tau) u_{\tau}$ is the unique weak solution of (1.1) with initial datum $u_{\tau}$ at time $\tau$; moreover, the process $\{U(t, \tau)\}_{t \geq \tau}$ is continuous in $L^{2}(\Omega)$ and is norm-to-weak continuous in $L^{p}(\Omega)$ and $\mathcal{D}_{0}^{1}(\Omega, \sigma)$, and has a pullback attractor $\mathcal{A}_{0}=\left\{A_{0}(t): t \in \mathbb{R}\right\}$ in $\mathcal{D}_{0}^{1}(\Omega, \sigma) \cap L^{p}(\Omega)$.

In this section, under the additional condition (H4) of the external force $g$, we will show that the pullback attractor is in fact in $\mathcal{D}_{0}^{2}(\Omega, \sigma) \cap L^{2 p-2}(\Omega)$.

Lemma 3.1. Assume that (H1)-(H3) hold. Then for any $t \in \mathbb{R}$, any bounded set $D$ of $L^{2}(\Omega)$, there exists $\tau_{0} \leq t-1$ such that

$$
\left|u_{t}(t)\right|_{2}^{2} \leq C\left(1+e^{-\lambda_{1} t} \int_{-\infty}^{t} e^{\lambda_{1} s}\left(|g(s)|_{2}^{2}+\left|g^{\prime}(s)\right|_{2}^{2}\right) d s\right),
$$

for any $\tau \leq \tau_{0}$ and any $u_{\tau} \in D$, where $u_{t}(s)=\left.\frac{d}{d t}\left(U(t, \tau) u_{\tau}\right)\right|_{t=s}$.

Proof. Let $u_{\tau} \in D$, and denote $u(t)=U(t, \tau) u_{\tau}$. Multiplying (1.1) by $u$ in $L^{2}(\Omega)$, we get

$$
\frac{1}{2} \frac{d}{d t}|u|_{2}^{2}+\|u\|_{\mathcal{D}_{0}^{1}(\Omega, \sigma)}^{2}+\int_{\Omega} f(u) u d x=\int_{\Omega} g(t) u d x \leq \frac{1}{\lambda_{1}}|g(t)|_{2}^{2}+\frac{\lambda_{1}}{4}|u|_{2}^{2} .
$$

From (H2) we have

$$
\int_{\Omega} f(u) u d x \geq C_{1} \int_{\Omega}|u|^{p} d x-C_{2}|\Omega| \geq C\left(2 \int_{\Omega} F(u) d x-1\right) .
$$


Using the inequality $\|u\|_{\mathcal{D}_{0}^{1}(\Omega, \sigma)}^{2} \geq \lambda_{1}|u|_{2}^{2}$ and (3.3), we get from (3.2),

$$
\frac{d}{d t}|u|_{2}^{2}+\lambda_{1}|u|_{2}^{2}+C\left(\|u\|_{\mathcal{D}_{0}^{1}(\Omega, \sigma)}^{2}+2 \int_{\Omega} F(u) d x\right) \leq C\left(1+|g(t)|_{2}^{2}\right)
$$

thus

$$
\frac{d}{d t}\left(e^{\lambda_{1} t}|u(t)|_{2}^{2}\right)+C e^{\lambda_{1} t}\left(\|u(t)\|_{\mathcal{D}_{0}^{1}(\Omega)}^{2}+2 \int_{\Omega} F(u(t)) d x\right) \leq C\left(e^{\lambda_{1} t}+e^{\lambda_{1} t}|g(t)|_{2}^{2}\right) .
$$

Integrating (3.5) from $\tau$ to $s, \tau \leq s \leq t-1$ in particular, we have

$$
e^{\lambda_{1} s}|u(s)|_{2}^{2} \leq e^{\lambda_{1} \tau}\left|u_{\tau}\right|_{2}^{2}+C\left(e^{\lambda_{1} s}+\int_{\tau}^{s} e^{\lambda_{1} r}|g(r)|_{2}^{2} d r\right)
$$

On the other hand, integrating (3.5) from $s$ to $s+1$ and applying (3.6), we get

$$
\begin{aligned}
\int_{s}^{s+1} e^{\lambda_{1} r} & \left(\|u(r)\|_{\mathcal{D}_{0}^{1}(\Omega, \sigma)}^{2}+2 \int_{\Omega} F(u(r)) d x\right) d r \\
& \leq C e^{\lambda_{1} s}|u(s)|_{2}^{2}+C \int_{s}^{s+1}\left(e^{\lambda_{1} r}+e^{\lambda_{1} r}|g(r)|_{2}^{2}\right) d r \\
& \leq C\left(e^{\lambda_{1} \tau}\left|u_{\tau}\right|_{2}^{2}+e^{\lambda_{1} t}+\int_{\tau}^{t} e^{\lambda_{1} r}|g(r)|_{2}^{2} d r\right) .
\end{aligned}
$$

Multiplying (1.1) by $u_{t}$ in $L^{2}(\Omega)$, we see that

$$
\left|u_{t}\right|_{2}^{2}+\frac{1}{2} \frac{d}{d t}\left(\|u\|_{\mathcal{D}_{0}^{1}(\Omega, \sigma)}^{2}+2 \int_{\Omega} F(u) d x\right)=\left(g(t), u_{t}\right) \leq \frac{1}{2}|g(t)|_{2}^{2}+\frac{1}{2}\left|u_{t}\right|_{2}^{2}
$$

Hence,

$$
\begin{aligned}
e^{\lambda_{1} t}\left|u_{t}(t)\right|_{2}^{2} & +\frac{d}{d t}\left[e^{\lambda_{1} t}\left(\|u(t)\|_{\mathcal{D}_{0}^{1}(\Omega, \sigma)}^{2}+2 \int_{\Omega} F(u(t)) d x\right)\right] \\
& \leq \lambda_{1} e^{\lambda_{1} t}\left(\|u(t)\|_{\mathcal{D}_{0}^{1}(\Omega, \sigma)}^{2}+2 \int_{\Omega} F(u(t)) d x\right)+e^{\lambda_{1} t}|g(t)|_{2}^{2} .
\end{aligned}
$$

Combining (3.7) and (3.9) and applying the uniform Gronwall inequality, we get

$$
e^{\lambda_{1} t}\left(\|u(t)\|_{\mathcal{D}_{0}^{1}(\Omega, \sigma)}^{2}+2 \int_{\Omega} F(u(t)) d x\right) \leq C\left(e^{\lambda_{1} \tau}\left|u_{\tau}\right|_{2}^{2}+e^{\lambda_{1} t}+\int_{\tau}^{t} e^{\lambda_{1} r}|g(r)|_{2}^{2} d r\right) .
$$


Integrating (3.9) from $r$ to $r+1, r \in[\tau, t-1]$ and using (3.7) and (3.10) in particular, we find that

$$
\begin{aligned}
& \int_{r}^{r+1} e^{\lambda_{1} s}|v|_{2}^{2} d s \\
& \leq e^{\lambda_{1} r}\left(\|u(r)\|_{\mathcal{D}_{0}^{1}(\Omega, \sigma)}^{2}+2 \int_{\Omega} F(u(r)) d x\right) \\
& \quad+\lambda_{1} \int_{r}^{r+1} e^{\lambda_{1} s}\left(\|u(s)\|_{\mathcal{D}_{0}^{1}(\Omega, \sigma)}^{2}+2 \int_{\Omega} F(u(s)) d x\right) d s+\int_{r}^{r+1} e^{\lambda_{1} s}|g(s)|_{2}^{2} d s \\
& \leq C\left(e^{\lambda_{1} \tau}\left|u_{\tau}\right|_{2}^{2}+e^{\lambda_{1} t}+\int_{-\infty}^{t} e^{\lambda_{1} s}|g(s)|_{2}^{2} d s\right) .
\end{aligned}
$$

On the other hand, differentiating equation (1.1) and denoting $v=u_{t}$, we have

$$
v_{t}-\operatorname{div}(\sigma \nabla v)+f^{\prime}(u) v=g^{\prime}(t) .
$$

Taking the inner product of (3.12) with $v$ in $L^{2}(\Omega)$, we get

$$
\frac{1}{2} \frac{d}{d t}|v|_{2}^{2}+\|v\|_{\mathcal{D}_{0}^{1}(\Omega, \sigma)}^{2}+\left(f^{\prime}(u) v, v\right)=\left(g^{\prime}(t), v\right) .
$$

Using (1.4) and Young's inequality, after some computations we see that

$$
\frac{d}{d t}\left(e^{\lambda_{1} t}|v|_{2}^{2}\right)+2 e^{\lambda_{1} t}\|v\|_{\mathcal{D}_{0}^{1}(\Omega, \sigma)}^{2} \leq C e^{\lambda_{1} t}|v|_{2}^{2}+C e^{\lambda_{1} t}\left|g^{\prime}(t)\right|_{2}^{2}
$$

Combining (3.14) and (3.11), and using the uniform Gronwall inequality, we obtain

$$
e^{\lambda_{1} t}|v(t)|_{2}^{2} \leq C\left(e^{\lambda_{1} \tau}\left|u_{\tau}\right|_{2}^{2}+e^{\lambda_{1} t}+\int_{-\infty}^{t} e^{\lambda_{1} s}\left(|g(s)|_{2}^{2}+\left|g^{\prime}(s)\right|_{2}^{2}\right) d s\right) .
$$

This completes the proof because $e^{\lambda_{1} \tau}\left|u_{\tau}\right|_{2}^{2} \rightarrow 0$ as $\tau \rightarrow-\infty$.

LEMMA 3.2. The process $\{U(t, \tau)\}$ associated to problem (1.1) has a family of pullback absorbing sets in $L^{2 p-2}(\Omega) \cap \mathcal{D}_{0}^{2}(\Omega, \sigma)$.

Proof. Multiplying the first equation in (1.1) by $|u|^{p-2} u$ and integrating over $\Omega$, we get

$$
\int_{\Omega} u_{t}|u|^{p-2} u d x+(p-1) \int_{\Omega} \sigma(x)|\nabla u|^{2}|u|^{p-2} d x+\int_{\Omega} f(u)|u|^{p-2} u d x=\int_{\Omega} g(t)|u|^{p-2} u d x .
$$

Using (1.2) and Young's inequality, we have

$$
\int_{\Omega} f(u)|u|^{p-2} u d x \geq \int_{\Omega}\left(C_{1}|u|^{p}-C_{2}\right)|u|^{p-2} d x \geq C_{1}\|u\|_{L^{2 p-2}(\Omega)}^{2 p-2}-C_{2}\|u\|_{L^{p}(\Omega)}^{p}-C_{3} .
$$

By the Cauchy inequality, we see that

$$
-\int_{\Omega} u_{t}|u|^{p-2} u d x \leq \frac{C_{1}}{4}\|u\|_{L^{2 p-2}(\Omega)}^{2 p-2}+\frac{1}{C_{1}}\left|u_{t}\right|_{2}^{2},
$$


and

$$
\int_{\Omega} g(t)|u|^{p-2} u d x \leq \frac{C_{1}}{4}\|u\|_{L^{2 p-2}(\Omega)}^{2 p-2}+\frac{1}{4}|g(t)|_{2}^{2} .
$$

It follows from (3.16)-(3.19) that

$$
\|u\|_{L^{2 p-2}(\Omega)}^{2 p-2} \leq C\left(1+\left|u_{t}\right|_{2}^{2}+\|u\|_{L^{p}(\Omega)}^{p}+|g(t)|_{2}^{2}\right) .
$$

To obtain estimates in $\mathcal{D}_{0}^{2}(\Omega, \sigma)$, we multiply equation (1.1) by $-\operatorname{div}(\sigma(x) \nabla u)$ and use the fact that $f(0)=0$ to obtain

$$
\|u\|_{\mathcal{D}_{0}^{2}(\Omega, \sigma)}^{2}=\int_{\Omega} u_{t} \operatorname{div}(\sigma(x) \nabla u) d x-\int_{\Omega} f^{\prime}(u) \sigma(x)|\nabla u|^{2} d x-\int_{\Omega} g(t) \operatorname{div}(\sigma(x) \nabla u) d x .
$$

Using the hypothesis $f^{\prime}(u) \geq-\ell$ and the Cauchy inequality, from (3.21) we have

$$
\|u\|_{\mathcal{D}_{0}^{2}(\Omega, \sigma)}^{2} \leq 2\left(\left|u_{t}\right|_{2}^{2}+\ell\|u\|_{\mathcal{D}_{0}^{1}(\Omega, \sigma)}^{2}+|g(t)|_{2}^{2}\right) .
$$

Taking into account (3.10) and (3.1), the relations (3.20) and (3.22) complete the proof.

REMARK 3.1. By Proposition 2.3, from Lemma 3.2 we can deduce that the process $U(t, \tau)$ associated to problem (1.1) is norm-to-weak continuous in $L^{2 p-2}(\Omega)$ and $\mathcal{D}_{0}^{2}(\Omega, \sigma)$.

We now derive some estimates for the time derivatives of $u$ by the well-known bootstrap technique. These estimates are useful for establishing pullback asymptotic $a$ priori estimates in $L^{2 p-2}(\Omega)$.

LemmA 3.3. For any $t \in \mathbb{R}$, any $2 \leq r<\infty$ and any bounded set $B \subset L^{2}(\Omega)$, there exists $\tau_{0}$ such that

$$
\int_{\Omega}\left|u_{t}(t)\right|^{r} d x \leq M, \text { for all } \tau \leq \tau_{0}, u_{\tau} \in B
$$

where $M$ depends on $t$ and $r$, but not on $u_{\tau}$ and $\tau$, and $u_{t}(s)=\left.\frac{d}{d t}\left(U(t, \tau) u_{\tau}\right)\right|_{t=s}$.

Proof. We will prove the lemma by an induction.

Putting $\beta=\frac{N}{N-2+\alpha}>1$. We will prove that for $k=0,1,2, \ldots$, there exist $\tau_{k}$ and $M_{k}(t)$ such that

$$
e^{\lambda_{1} t} \int_{\Omega}|v(t)|^{2 \beta^{k}} d x \leq M_{k}(t) \text { for any } u_{\tau} \in B, \tau \leq \tau_{k},
$$

and

$$
\int_{t}^{t+1}\left(e^{\lambda_{1} r} \int_{\Omega}|v(r)|^{2 \beta^{k+1}} d x\right)^{\frac{1}{\beta}} d r \leq M_{k}(t) \text { for any } u_{\tau} \in B, \tau \leq \tau_{k},
$$

where $\tau_{k}$ depends on $k$ and $B ; M_{k}(t)$ depends only on $k$.

For $k=0,\left(P_{0}\right)$ follows from (3.15). Integrating (3.14) from $t$ to $t+1$ and using the facts that $\frac{1}{\beta}<1$ and $\mathcal{D}_{0}^{1}(\Omega, \sigma) \hookrightarrow L^{2 \beta}(\Omega)$ continuously, we get $\left(Q_{0}\right)$. 
Assuming that $\left(P_{k}\right)$ and $\left(Q_{k}\right)$ hold, we prove that $\left(P_{k+1}\right)$ and $\left(Q_{k+1}\right)$ also hold. Multiplying (3.12) by $|v|^{2 \beta^{k+1}-2} v$ and integrating over $\Omega$, we obtain

$$
C \frac{d}{d t} \int_{\Omega}|v|^{2 \beta^{k+1}} d x+C \int_{\Omega} \sigma(x)\left|\nabla\left(v^{\beta^{k+1}}\right)\right|^{2} d x \leq \ell \int_{\Omega}|v|^{2 \beta^{k+1}} d x+\left(g^{\prime}(t),|v|^{2 \beta^{k+1}-2} v\right) .
$$

Using the imbedding $\mathcal{D}_{0}^{1}(\Omega, \sigma) \hookrightarrow L^{2 \beta}(\Omega)$ once again, we get

$$
\int_{\Omega} \sigma(x)\left|\nabla\left(v^{\beta^{k+1}}\right)\right|^{2} d x \geq\left\|v^{\beta^{k+1}}\right\|_{L^{2 \beta}(\Omega)}^{2}=\left(\int_{\Omega}|v|^{2 \beta^{k+2}} d x\right)^{\frac{1}{\beta}} .
$$

Combining Hölder's and Young's inequalities, we see that

$$
\begin{aligned}
\int_{\Omega} g^{\prime}(t)|v|^{2 \beta^{k+1}-2} v d x & \leq\left(\int_{\Omega}\left|g^{\prime}(t)\right|^{m} d x\right)^{1 / m}\left(\int_{\Omega}|v|^{\left(2 \beta^{k+1}-1\right) n} d x\right)^{1 / n} \\
& \leq \frac{\left(\int_{\Omega}\left|g^{\prime}(t)\right|^{m} d x\right)^{m^{\prime} / m}}{m^{\prime}}+\frac{\left(\int_{\Omega}|v|^{\left(2 \beta^{k+1}-1\right) n} d x\right)^{n^{\prime} / n}}{n^{\prime}}
\end{aligned}
$$

where $\frac{1}{m}+\frac{1}{n}=\frac{1}{m^{\prime}}+\frac{1}{n^{\prime}}=1$. Choose $n, n^{\prime}$ such that

$$
\left(2 \beta^{k+1}-1\right) n=2 \beta^{k+2} \text { and } \frac{n^{\prime}}{n}=\frac{1}{\beta},
$$

that is,

$$
n=\frac{2 \beta^{k+2}}{2 \beta^{k+1}-1} \text { and } n^{\prime}=\frac{2 \beta^{k+1}}{2 \beta^{k+1}-1}
$$

Hence,

$$
m=\frac{n}{n-1}=\frac{2 \beta^{k+2}}{2 \beta^{k+2}-2 \beta^{k+1}+1} \text { and } m^{\prime}=2 \beta^{k+1} .
$$

Then from (3.26), we infer that

$$
\int_{\Omega} g^{\prime}(t)|v|^{2 \beta^{k+1}-2} v d x \leq \frac{1}{m^{\prime}}\left\|g^{\prime}(t)\right\|_{L^{m}(\Omega)}^{m^{\prime}}+\frac{1}{n^{\prime}}\left(\int_{\Omega}|v|^{2 \beta^{k+2}} d x\right)^{\frac{1}{\beta}} .
$$

Applying (3.25) and (3.28) in (3.24), we find that

$$
\begin{aligned}
& \frac{d}{d t}\left(e^{\lambda_{1} t} \int_{\Omega}|v|^{2 \beta^{k+1}} d x\right)+C e^{\lambda_{1} t}\left(\int_{\Omega}|v|^{2 \beta^{k+2}} d x\right)^{\frac{1}{\beta}} \\
\leq & C e^{\lambda_{1} t} \int_{\Omega}|v|^{2 \beta^{k+1}} d x+C e^{\lambda_{1} t}\left\|g^{\prime}(t)\right\|_{L^{m}(\Omega)}^{m^{\prime}} .
\end{aligned}
$$

Combining $\left(Q_{k}\right)$ and (3.29), using the uniform Gronwall inequality and taking into account assumption (H4), we get $\left(P_{k+1}\right)$. On the other hand, integrating (3.29) from $t$ to $t+1$ we find $\left(Q_{k+1}\right)$. Now, since $\beta>1$, taking $k \geq \log _{\beta} \frac{r}{2}$ we get the desired estimate. 
In what follows, we will use the following lemma.

Lemma 3.4 [13, Lemma 3.6]. If there exists $\sigma>0$ such that $\int_{-\infty}^{t} e^{\sigma s}|\varphi(s)|^{2} d s<\infty$, for all $t \in \mathbb{R}$, then

$$
\lim _{\gamma \rightarrow+\infty} \int_{-\infty}^{t} e^{-\gamma(t-s)}|\varphi(s)|^{2} d s=0, \quad t \in \mathbb{R}
$$

Let $H_{m}=\operatorname{span}\left\{e_{1}, e_{2}, \ldots, e_{m}\right\}$ in $L^{2}(\Omega)$ and $P_{m}: L^{2}(\Omega) \rightarrow H_{m}$ be the orthogonal projection, where $\left\{e_{i}\right\}_{i=1}^{\infty}$ are eigenvectors of the operator $A=-\operatorname{div}(\sigma(x) \nabla)$. For any $u \in L^{2}(\Omega)$, then $u$ has a unique decomposition

$$
u=P_{m} u+\left(I-P_{m}\right) u=u_{1}+u_{2} .
$$

Lemma 3.5. For any $t \in \mathbb{R}$, any bounded set $B$ of $L^{2}(\Omega)$ and any $\varepsilon$, there exist $\tau_{0}(t, B, \varepsilon)$ and $m_{0} \in \mathbb{N}$ such that

$$
\left|\left(I-P_{m}\right) v\right|_{2}^{2}<\varepsilon, \forall \tau \leq \tau_{0}, \forall u_{\tau} \in B \text { and } m \geq m_{0},
$$

where $v=u_{t}$.

Proof. Multiplying (3.12) by $v_{2}=\left(I-P_{m}\right) v$ and integrating over $\Omega$, using the inequality $\left\|v_{2}\right\|_{\mathcal{D}_{0}^{1}(\Omega, \sigma)}^{2} \geq \lambda_{m}\left|v_{2}\right|_{2}^{2}$ and the Cauchy inequality, we get

$$
\frac{d}{d t}\left|v_{2}\right|_{2}^{2}+\lambda_{m}\left|v_{2}\right|_{2}^{2} \leq C \int_{\Omega}\left|f^{\prime}(u) v\right|^{2} d x+C\left|g^{\prime}(t)\right|_{2}^{2} .
$$

We multiply (3.32) by $e^{\lambda_{m} t}$ and use assumption (1.3) to get

$$
\frac{d}{d t}\left(e^{\lambda_{m} t}\left|v_{2}\right|_{2}^{2}\right) \leq C e^{\lambda_{m} t} \int_{\Omega}|u|^{2(p-2)}|v|^{2} d x+C e^{\lambda_{m} t}\left|g^{\prime}(t)\right|_{2}^{2} .
$$

Integrating (3.33) from $s$ to $t$,

$$
\begin{aligned}
e^{\lambda_{m} t}\left|v_{2}(t)\right|_{2}^{2} & \leq e^{\lambda_{m} s}\left|v_{2}(s)\right|_{2}^{2}+C \int_{s}^{t} e^{\lambda_{m} r} \int_{\Omega}|u|^{2(p-2)}|v|^{2} d x d r+C \int_{s}^{t} e^{\lambda_{m} r}\left|g^{\prime}(r)\right|_{2}^{2} d r \\
& \leq e^{\lambda_{m} s}|v(s)|_{2}^{2}+C \int_{-\infty}^{t} e^{\lambda_{m} r} \int_{\Omega}|u|^{2(p-2)}|v|^{2} d x d r+C \int_{-\infty}^{t} e^{\lambda_{m} r}\left|g^{\prime}(r)\right|_{2}^{2} d r
\end{aligned}
$$

Now integrating (3.34) with respect to $s$ from $\tau$ to $t$, we infer that

$$
\begin{aligned}
(t-\tau) e^{\lambda_{m} t}\left|v_{2}(t)\right|_{2}^{2} \leq & \int_{\tau}^{t} e^{\lambda_{m} s}|v(s)|_{2}^{2} d s+C(t-\tau) \int_{-\infty}^{t} e^{\lambda_{m} r} \int_{\Omega}|u|^{2(p-2)}|v|^{2} d x d r \\
& +C(t-\tau) \int_{-\infty}^{t} e^{\lambda_{m} r}\left|g^{\prime}(r)\right|_{2}^{2} d r
\end{aligned}
$$

thus

$$
\begin{aligned}
\left|v_{2}(t)\right|_{2}^{2} \leq & \frac{1}{t-\tau} \int_{-\infty}^{t} e^{-\lambda_{m}(t-s)}|v(s)|_{2}^{2} d s+C \int_{-\infty}^{t} e^{-\lambda_{m}(t-r)} \int_{\Omega}|u|^{2(p-2)}|v|^{2} d x d r \\
& +C \int_{-\infty}^{t} e^{-\lambda_{m}(t-r)}\left|g^{\prime}(r)\right|_{2}^{2} d r
\end{aligned}
$$


By Lemma 3.4 and since $\lambda_{m} \rightarrow+\infty$ as $m \rightarrow+\infty$, there exist $\tau_{1}$ and $m_{1}$ such that

$$
\frac{1}{t-\tau} \int_{-\infty}^{t} e^{-\lambda_{m}(t-s)}|v(s)|_{2}^{2} d s<\frac{\varepsilon}{3}
$$

and

$$
C \int_{-\infty}^{t} e^{-\lambda_{m}(t-r)}\left|g^{\prime}(r)\right|_{2}^{2} d r<\frac{\varepsilon}{3},
$$

for all $\tau \leq \tau_{1}$ and $m \geq m_{1}$. For the second term on the right-hand side of (3.36), using Hölder's inequality we have

$$
\begin{aligned}
& \int_{-\infty}^{t} e^{-\lambda_{m}(t-r)} \int_{\Omega}|u|^{2(p-2)}|v|^{2} d x d r \\
& \quad \leq \int_{-\infty}^{t}\left(\int_{\Omega} e^{-\frac{p-1}{p-2} \lambda_{m}(t-r)}|u|^{2 p-2} d x\right)^{\frac{p-2}{p-1}}\left(\int_{\Omega} e^{-(p-1) \lambda_{m}(t-r)}|v|^{2 p-2} d x\right)^{\frac{1}{p-1}} d r \\
& \quad \leq\left(\int_{-\infty}^{t} e^{-\frac{p-1}{p-2} \lambda_{m}(t-r)}\|u\|_{L^{2 p-2}(\Omega)}^{2 p-2} d r\right)^{\frac{p-2}{p-1}}\left(\int_{-\infty}^{t} e^{-(p-1) \lambda_{m}(t-r)} \int_{\Omega}|v|^{2 p-2} d x d r\right)^{\frac{1}{p-1}} .
\end{aligned}
$$

Using Lemmas 3.2-3.4, there exist $\tau_{2}$ and $m_{2} \in \mathbb{N}$ such that

$$
C \int_{-\infty}^{t} e^{-\lambda_{m}(t-r)} \int_{\Omega}|u|^{2(p-2)}|v|^{2} d x d r<\frac{\varepsilon}{3}, \text { for all } \tau \leq \tau_{0}, m \geq m_{2} .
$$

Let $\tau_{0}=\min \left\{\tau_{1}, \tau_{2}\right\}$ and $m_{0}=\max \left\{m_{1}, m_{2}\right\}$, from (3.36), taking into account (3.37), (3.38) and (3.40) we obtain (3.31).

LeMma 3.6 [14]. Let $B$ be a bounded subset in $L^{q}(\Omega)(q \geq 1)$. If B has a finite $\varepsilon-n e t$ in $L^{q}(\Omega)$, then there exists a number $M=M(B, \varepsilon)$ such that for any $u \in B$, the following estimate holds

$$
\int_{\Omega(|u| \geq M)}|u|^{q} d x<\varepsilon
$$

where $\Omega(|u| \geq M)=\{x \in \Omega:|u| \geq M\}$.

Using Lemma 3.6 and taking into account Lemmas 3.1 and 3.5, we conclude that the set $\left\{u_{t}(s): s \leq t, u_{\tau} \in B\right\}$ has a finite $\varepsilon$-net in $L^{2}(\Omega)$. Therefore, we get the following result.

Lemma 3.7. For any $t \in \mathbb{R}$, any $B \subset L^{2}(\Omega)$ is bounded and any $\varepsilon>0$, there exist $\tau_{0} \leq t$ and $M_{0}>0$ such that

$$
\int_{\Omega(|u| \geq M)}\left|u_{t}(t)\right|^{2} d x<\varepsilon, \text { for all } \tau<\tau_{0}, M>M_{0}, u_{\tau} \in B .
$$

One can prove the following lemma by using arguments in [14] with a few modifications. 
Lemma 3.8 [14]. For any $t \in \mathbb{R}$, any bounded set $B \subset L^{2}(\Omega)$ and any $\varepsilon>0$, there exist $\tau_{0}$ and $M_{0}>0$ such that

$$
\operatorname{mes}(\Omega(u(t) \geq M))<\varepsilon, \text { for all } \tau \leq \tau_{0}, M \geq M_{0} \text { and } u_{\tau} \in B,
$$

where mes is the Lebesgue measure in $\mathbb{R}^{N}$.

LEMMA 3.9 [2]. Let $\{U(t, \tau)\}$ be a norm-to-weak continuous process in $L^{2}(\Omega)$ and $L^{q}(\Omega), q \geq 2$. Then $\{U(t, \tau)\}$ is pullback asymptotically compact in $L^{q}(\Omega)$ if

(i) $\{U(t, \tau)\}$ is pullback asymptotically compact in $L^{2}(\Omega)$, and

(ii) for any $t \in \mathbb{R}$, any bounded set $D \subset L^{2}(\Omega)$ and any $\varepsilon>0$, there exist $M>0$ and $\tau_{0} \leq t$ such that

$$
\sup _{\tau \leq \tau_{0}} \sup _{u_{\tau} \in D}\left(\int_{\Omega\left(\left|U(t, \tau) u_{\tau}\right| \geq M\right)}\left|U(t, \tau) u_{\tau}\right|^{q} d x\right) \leq C \varepsilon,
$$

where $C$ is independent of $M, \tau, u_{\tau}$ and $\varepsilon$.

We are now ready to prove the existence of a pullback attractor in $L^{2 p-2}(\Omega)$.

THEOREM 3.10. Assume that assumptions (H1)-(H4) hold. Then the process $\{U(t, \tau)\}$ associated to problem (1.1) possesses a pullback attractor $\mathcal{A}_{2 p-2}=\left\{A_{2 p-2}(t)\right.$ : $t \in \mathbb{R}\}$ in $L^{2 p-2}(\Omega)$.

Proof. Since $\{U(t, \tau)\}$ has a family of pullback absorbing sets in $L^{2 p-2}(\Omega)$, by Lemma 3.9, we only have to prove that for any $t \in \mathbb{R}$, any bounded set $B \subset L^{2}(\Omega)$ and any $\varepsilon>0$, there exist $\tau_{2} \leq t$ and $M_{2}>0$ such that

$$
\int_{\Omega(|u| \geq M)}|u|^{2 p-2} d x \leq C \varepsilon, \forall \tau \leq \tau_{2}, M \geq M_{2}, u_{\tau} \in B .
$$

Taking the inner product of $(1.1)$ with $(u-M)_{+}^{p-1}$ in $L^{2}(\Omega)$, where

$$
(u-M)_{+}= \begin{cases}u-M & \text { if } u \geq M \\ 0 & \text { otherwise }\end{cases}
$$

we have

$$
\begin{aligned}
\int_{\Omega} u_{t}(u-M)_{+}^{p-1} d x & +(p-1) \int_{\Omega} \sigma(x)|\nabla u|^{2}(u-M)_{+}^{p-2} d x \\
& +\int_{\Omega} f(u)(u-M)_{+}^{p-1} d x=\int_{\Omega} g(t)(u-M)_{+}^{p-1} d x .
\end{aligned}
$$

Some standard computations give that

$$
\begin{gathered}
\int_{\Omega} f(u)(u-M)_{+}^{p-1} d x \geq C_{0} \int_{\Omega(u \geq M)}|u|^{2 p-2} d x+C \int_{\Omega}|u|^{p} d x, \\
-\int_{\Omega} u_{t}(u-M)_{+}^{p-1} d x \leq \frac{C_{0}}{4} \int_{\Omega(u \geq M)}|u|^{2 p-2} d x+\frac{1}{C_{0}} \int_{\Omega(u \geq M)}\left|u_{t}\right|^{2} d x
\end{gathered}
$$


and

$$
\int_{\Omega} g(t)(u-M)_{+}^{p-1} \leq \frac{C_{0}}{4} \int_{\Omega(u \geq M)}|u|^{2 p-2} d x+\frac{1}{C_{0}} \int_{\Omega(u \geq M)}|g(t)|^{2} d x .
$$

Combining (3.43)-(3.46), we have

$$
\int_{\Omega(u \geq M)}|u|^{2 p-2} d x \leq C\left(\int_{\Omega(u \geq M)}\left|u_{t}\right|^{2} d x+\int_{\Omega(u \geq M)}|g(t)|^{2} d x+\int_{\Omega(u \geq M)}|u|^{p} d x\right) .
$$

Using Lemmas 3.7 and 3.8 and the fact that $\{U(t, \tau)\}$ has a pullback attractor in $L^{p}(\Omega)$, from (3.47) we can find $\tau_{0}$ and $M_{0}$ such that

$$
\int_{\Omega(u \geq M)}|u|^{2 p-2} d x<\varepsilon \text { for all } \tau \leq \tau_{0}, M \geq M_{0} .
$$

Repeating the above arguments with $\left|(u+M)_{-}\right|^{p-2}(u+M)_{-}$instead of $(u-M)_{+}^{p-1}$, we have

$$
\int_{\Omega(u \leq-M)}|u|^{2 p-2} d x<\varepsilon \text { for all } \tau \leq \tau_{1}, M \geq M_{1},
$$

for some $\tau_{1} \leq t$ and $M_{1}>0$, where

$$
(u+M)_{-}=\left\{\begin{array}{ll}
u+M & \text { if } u \leq-M \\
0 & \text { otherwise }
\end{array} .\right.
$$

Letting $\tau_{2}=\min \left\{\tau_{0}, \tau_{1}\right\}$ and $M_{2}=\max \left\{M_{0}, M_{1}\right\}$, we have

$$
\int_{\Omega\left(|u| \geq M_{2}\right)}|u|^{2 p-2}<C \varepsilon, \forall \tau \leq \tau_{2}, M \geq M_{2} .
$$

This completes the proof.

In order to prove the existence of a pullback attractor in $\mathcal{D}_{0}^{2}(\Omega, \sigma)$, we will use the '(PDC) condition' defined as follows.

Definition 3.1 [9]. Let $X$ be a Banach space. A process $U(t, \tau): L^{2}(\Omega) \rightarrow X$ is said to satisfy (PDC) condition in $X$ if for any $t \in \mathbb{R}$, any bounded subset $B \subset L^{2}(\Omega)$ and any $\varepsilon>0$, there exists $\tau_{0} \leq t$ and a finite dimensional subspace $X_{1}$ of $X$ such that

(i) $P\left(\bigcup_{\tau \leq \tau_{0}} U(t, \tau) B\right)$ is bounded in $X$, and

(ii) $\left\|\left(I_{X}-P\right) U(t, \tau) u_{\tau}\right\|_{X}<\varepsilon$, for all $\tau \leq \tau_{0}$ and $u_{\tau} \in B$, where $P: X \rightarrow X_{1}$ is a canonical projection and $I_{X}$ is the identity.

Lemma 3.11 [9]. If a process $\{U(t, \tau)\}$ satisfies (PDC) condition in $X$, then it is pullback asymptotically compact in $X$. Moreover, if $X$ is convex, then the converse is true.

Lemma 3.12 [14]. Assume that $f$ satisfies (H2). Then for any subset $A \subset L^{2 p-2}(\Omega)$, if $\kappa(A)<\varepsilon$ in $L^{2 p-2}(\Omega)$, we have

$$
\kappa(f(A))<C \varepsilon \text { in } L^{2}(\Omega)
$$


where $\kappa($.$) is the Kuratowski non-compactness measure in a Banach space X$ defined as

$$
\kappa(B)=\inf \{\delta>0: \text { B has a finite covering by balls in Xwith radii } \delta\} .
$$

THEOREM 3.13. Assume that assumptions (H1)-(H4) hold. Then the process $\{U(t, \tau)\}$ associated to problem (1.1) has a pullback attractor $\mathcal{A}=\{A(t): t \in \mathbb{R}\}$ in $\mathcal{D}_{0}^{2}(\Omega, \sigma)$.

Proof. We consider a complete trajectory $u(t)$ lying on the pullback attractor $\mathcal{A}_{2 p-2}$ in $L^{2 p-2}(\Omega)$ for $\{U(t, \tau)\}$, that is, $u(t) \in A_{2 p-2}(t)$ and $U(t, \tau) u(\tau)=u(t)$, for all $t \geq \tau$. Denoting $A=-\operatorname{div}(\sigma(x) \nabla)$ and multiplying $(1.1)$ by $A u_{2}=A\left(I-P_{m}\right) u=(I-$ $\left.P_{m}\right) A u$, we have

$$
\left(u_{t}, A u_{2}\right)+\left\|u_{2}\right\|_{\mathcal{D}_{0}^{2}(\Omega, \sigma)}^{2}+\int_{\Omega} f(u) A u_{2} d x=\left(g(t), A u_{2}\right) .
$$

Using the Cauchy inequality, we get

$$
\left\|u_{2}\right\|_{\mathcal{D}_{0}^{2}(\Omega, \sigma)}^{2} \leq C\left(\left|\left(I-P_{m}\right) u_{t}\right|_{2}^{2}+\left|\left(I-P_{m}\right) g(t)\right|_{2}^{2}+\int_{\Omega}(f(u))^{2} d x\right) .
$$

Thanks to Lemmas 3.5 and 3.12 and the fact that $g \in C_{\text {loc }}\left(\mathbb{R} ; L^{2}(\Omega)\right)$, we see that $\{U(t, \tau)\}$ satisfies the (PDC) condition in $\mathcal{D}_{0}^{2}(\Omega, \sigma)$. Now the result follows from Lemmas 3.2 and 3.11 .

4. Fractal dimension estimates of the pullback attractor. In this section, we will prove that the pullback attractor has a finite fractal dimension in $L^{2}(\Omega)$.

THEOREM 4.1. Assume that $f$ and $g$ satisfy conditions $(H 2)-(H 4)$, and that $g$ satisfies the following additional condition

$$
\int_{-\infty}^{0} \int_{-\infty}^{s} e^{\lambda_{1} r}\left(|g(r)|_{2}^{2}+\left|g^{\prime}(r)\right|_{2}^{2}\right) d r d s<+\infty
$$

Then the pullback attractor $\mathcal{A}$ of the process $U(t, \tau)$ associated to problem (1.1) has a finite fractal dimension in $L^{2}(\Omega)$, and

$$
\operatorname{dim}_{F}(A(t)) \leq m \log \left(1+\frac{8 e^{2 \ell}}{1-\delta}\right)\left(\log \frac{2}{1+\delta}\right)^{-1}, \forall t \in \mathbb{R},
$$

for some $\delta<1$ and some $m \in \mathbb{N}$.

Proof. According to (3.10), we easily see that there exists a uniform pullback absorbing set $\mathcal{B}_{0}$ of $\{U(t, \tau)\}$ in $\mathcal{D}_{0}^{1}(\Omega, \sigma)$. Now let $u(t)=U(t, \tau) u_{\tau}$ and $v(t)=U(t, \tau) v_{\tau}$ be solutions of problem (1.1) with initial data $u_{\tau}, v_{\tau} \in \mathcal{B}_{0}$ respectively.

Let $w(t)=u(t)-v(t)$. Then we have

$$
\frac{1}{2} \frac{d}{d t}|w(t)|_{2}^{2}+\|w(t)\|_{\mathcal{D}_{0}^{1}(\Omega, \sigma)}^{2}+(f(u)-f(v), w)=0 .
$$


Using (1.4), we get

$$
\frac{d}{d t}|w(t)|_{2}^{2} \leq 2 \ell|w(t)|_{2}^{2}
$$

thus

$$
|w(t)|_{2}^{2} \leq e^{2 \ell(t-\tau)}|w(\tau)|_{2}^{2}
$$

Let $w=P_{m} w+\left(I-P_{m}\right) w=w_{1}+w_{2}$, then

$$
\left|w_{1}(t)\right|_{2}^{2} \leq e^{2 \ell(t-\tau)}|w(\tau)|_{2}^{2} .
$$

Taking the inner product of (1.1) with $w_{2}$ in $L^{2}(\Omega)$, we obtain

$$
\frac{1}{2} \frac{d}{d t}\left|w_{2}(t)\right|_{2}^{2}+\left\|w_{2}(t)\right\|_{\mathcal{D}_{0}^{1}(\Omega, \sigma)}^{2}+\left(f(u)-f(v), w_{2}\right)=0 .
$$

Taking into account (1.2) and Hölder's inequality, we see that

$$
\begin{aligned}
\left|\left(f(u)-f(v), w_{2}\right)\right| & \leq\left(\int_{\Omega}|f(u)-f(v)|^{2} d x\right)^{1 / 2}\left(\int_{\Omega}\left|w_{2}\right|^{2} d x\right)^{1 / 2} \\
& \leq C\left(\int_{\Omega}\left(1+|u|^{p-1}+|v|^{p-1}\right)^{2} d x\right)^{1 / 2}\left(\int_{\Omega}\left|w_{2}\right|^{2} d x\right)^{1 / 2} \\
& \leq C\left(1+\|u\|_{L^{2 p-2}(\Omega)}^{2 p-2}+\|v\|_{L^{2 p-2}(\Omega)}^{2 p-2}\right)^{1 / 2}|w|_{2} .
\end{aligned}
$$

Combining (4.6), (4.7) and noticing that $\left\|w_{2}\right\|_{\mathcal{D}_{0}^{1}(\Omega, \sigma)}^{2} \geq \lambda_{m}\left|w_{2}\right|_{2}^{2}$, we have

$$
\frac{d}{d t}\left|w_{2}(t)\right|_{2}^{2}+2 \lambda_{m}\left|w_{2}(t)\right|_{2}^{2} \leq C\left(1+\|u\|_{L^{2 p-2}(\Omega)}^{2 p-2}+\|v\|_{L^{2 p-2}(\Omega)}^{2 p-2}\right)^{1 / 2}|w|_{2} .
$$

Using Gronwall's lemma and (3.20) we conclude that

$$
\begin{aligned}
\left|w_{2}(t)\right|_{2}^{2} \leq & e^{-\lambda_{m}(t-\tau)}|w(\tau)|_{2}^{2} \\
& +C e^{-\lambda_{m} t} \int_{\tau}^{t} e^{\lambda_{m} s}\left(1+\|u(s)\|_{L^{2 p-2}(\Omega)}^{2 p-2}+\|v(s)\|_{L^{2 p-2}(\Omega)}^{2 p-2}\right)^{1 / 2}|w(s)|_{2} d s \\
\leq & e^{-\lambda_{m}(t-\tau)}|w(\tau)|_{2}^{2}+C \int_{\tau}^{t} e^{-\lambda_{m}(t-s)}\left(1+\|u(s)\|_{L^{p}(\Omega)}^{p}+\|v(s)\|_{L^{p}(\Omega)}^{p}\right. \\
& \left.+\left|u_{t}(s)\right|_{2}^{2}+\left|v_{t}(s)\right|_{2}^{2}+|g(s)|_{2}^{2}\right)|w(s)|_{2} d s \leq e^{-\lambda_{m}(t-\tau)}|w(\tau)|_{2}^{2} \\
& +C e^{\ell(t-\tau)}|w(\tau)|_{2} \int_{\tau}^{t} e^{-\lambda_{m}(t-s)}\left(1+\|u(s)\|_{L^{p}(\Omega)}^{p}+\|v(s)\|_{L^{p}(\Omega)}^{p}\right. \\
& \left.+\left|u_{t}(s)\right|_{2}^{2}+\left|v_{t}(s)\right|_{2}^{2}+|g(s)|_{2}^{2}\right) d s .
\end{aligned}
$$

It is easy to see from (3.10), (4.1) and (3.15) that

$$
\int_{-\infty}^{t} e^{\lambda_{1} s}\left(\|u(s)\|_{L^{p}(\Omega)}^{p}+\|v(s)\|_{L^{p}(\Omega)}^{p}+\left|u_{t}(s)\right|_{2}^{2}+\left|v_{t}(s)\right|_{2}^{2}\right) d s<+\infty, \quad \forall t \in \mathbb{R} .
$$


Thus, using Lemma 3.4 we get

$$
\lim _{m \rightarrow \infty} \int_{\tau}^{t} e^{-\lambda_{m}(t-s)}\left(1+\|u(s)\|_{L^{p}(\Omega)}^{p}+\|v(s)\|_{L^{p}(\Omega)}^{p}+\left|u_{t}(s)\right|_{2}^{2}+\left|v_{t}(s)\right|_{2}^{2}+|g(s)|_{2}^{2}\right) d s=0 .
$$

Let $T_{0}=t-\tau=1$, by combining (4.10) with (4.9), we get $m$ large enough so that

$$
\left|w_{2}(t)\right|_{2}^{2} \leq \delta|w(\tau)|_{2}
$$

with $\delta<1$. Thus, from (4.5) and (4.11), using Theorem 2.5 we get the result.

5. Some further remarks. We have just proved the existence of pullback attractors $\mathcal{A}=\{A(t): t \in \mathbb{R}\}$ of 'fixed' bounded sets for problem (1.1). It is noted that the pullback attractors obtained in Theorems 3.10 and 3.13 are the same object and equal to the pullback attractor obtained in [2]. On the other hand, several authors use the concept of attraction in a universe $\mathcal{D}$ not only composed by 'fixed' sets, but also moving in time, which usually appears in applications and is defined in terms of a tempered condition (see e.g. [4]). Here $\mathcal{D}$ is a non-empty class of bounded sets parameterized in time $\hat{D}=\{D(t): t \in \mathbb{R}\}$ with a tempered condition on their growth in time. When $\mathcal{D}$ is the universe of all constant bounded subsets, the pullback $\mathcal{D}$-attractor is equal to the pullback attractors of 'fixed' bounded sets. We now give some remarks about the relationship between these two concepts of pullback attractors for problem (1.1) base on the abstract results obtained in [11].

Firstly, let $\mathcal{R}$ be the set of all functions $r: \mathbb{R} \rightarrow(0,+\infty)$ satisfying the tempered condition $\lim _{t \rightarrow-\infty} e^{\lambda_{1} t} r^{2}(t)=0$, and denote by $\mathcal{D}$ the class of all families $\hat{\mathcal{D}}=\{D(t)$ : $t \in \mathbb{R}\}$ such that $D(t) \subset \bar{B}(r(t))$ for some $r \in \mathcal{R}$, where $\bar{B}(r(t))$ is the closed ball in $\mathcal{D}_{0}^{2}(\Omega, \sigma) \cap L^{2 p-2}(\Omega)$ with radius $r(t)$. Using this universe $\mathcal{D}$, one may establish the existence of a pullback $\mathcal{D}$-attractor $\mathcal{A}_{\mathcal{D}}=\left\{A_{\mathcal{D}}(t): t \in \mathbb{R}\right\}$ for problem (1.1) by arguments similar to the ones given in Section 3. As a corollary of the results in [11], this pullback $\mathcal{D}$-attractor contains the pullback attractor obtained in Theorems 3.10 and 3.13. Moreover, also using the abstract results in [11], one can see that if $g$ satisfies an additional hypothesis, namely $\sup _{t \leq T}|g(t)|_{2}^{2}<\infty$ for some $T$, then these two pullback attractors are coincident for all $t \leq T$, that is $A_{\mathcal{D}}(t)=A(t)$ for all $t \leq T$.

Acknowledgements. This work was supported by Vietnam's National Foundation for Science and Technology Development (NAFOSTED) under Project 101.01-2010.05.

\section{REFERENCES}

1. M. Anguiano, T. Caraballo and J. Real, $H^{2}$-boundedness of the pullback attractor for a non-autonomous reaction-diffusion equation, Nonlinear Anal. 72 (2010), 876-880.

2. C. T. Anh and T. Q. Bao, Pullback attractors for a non-autonomous semilinear degenerate parabolic equation, Glasgow Math. J. 52 (2010), 537-554.

3. P. Caldiroli and R. Musina, On a variational degenerate elliptic problem, Nonlinear Diff. Equ. Appl. 7 (2000), 187-199.

4. T. Caraballo, G. Lukaszewicz and J. Real, Pullback attractors for asymptotically compact nonautonomous dynamical systems, Nonlinear Anal. 64 (2006), 484-498. 
5. R. Dautray and J. L. Lions, Mathematical analysis and numerical methods for science and technology, Vol. I: Physical origins and classical methods (Springer-Verlag, Berlin, Germany, 1985).

6. N. I. Karachalios and N. B. Zographopoulos, Convergence towards attractors for a degenerate Ginzburg-Landau equation, Z. Angew. Math. Phys. 56 (2005), 11-30.

7. N. I. Karachalios and N. B. Zographopoulos, On the dynamics of a degenerate parabolic equation: Global bifurcation of stationary states and convergence, Calc. Var. Partial Differ. Equ. 25(3) (2006), 361-393.

8. Y. Li, S. Wang and J. Wei, Finite fractal dimension of pullback attractors and application to non-autonomous reaction diffusion equations, Appl. Math. E-Notes 10 (2010), 19-26.

9. Y. Li and C. K. Zhong, Pullback attractors for the norm-to-weak continuous process and application to the nonautonomous reaction-diffusion equations, Appl. Math. Comp. 190 (2007), 1020-1029.

10. G. Łukaszewicz, On pullback attractors in $L^{p}$ for nonautonomous reaction-diffusion equations, Nonlinear Anal. 73 (2010), 350-357.

11. P. Marin-Rubio and J. Real, On the relation between two different concepts of pullback attractors for non-autonomous dynamical systems, Nonlinear Anal. 71 (2009), 3956-3963.

12. H. Song and C. K. Zhong, Attractor of non-autonomous reaction-diffusion equation in $L^{p}$, Nonlinear Anal. 68 (2008), 1890-1897.

13. Y. Wang and C. K. Zhong, On the existence of pullback attractors for non-autonomous reaction-diffusion equations, Dyn. Syst. 23(1) (2008), 1-16.

14. C. K. Zhong, M. H. Yang and C. Y. Sun, The existence of global attractors for the normto-weak continuous semigroup and application to the nonlinear reaction-diffusion equations, J. Differ. Equ. 15 (2006), 367-399. 